\title{
Mapping Template Heart Models to Patient Data Using Image Registration
}

\author{
Marcin Wierzbicki $^{1,2}$, Maria Drangova ${ }^{1,2}$, Gerard Guiraudon ${ }^{1,2,3}$, and Terry Peters ${ }^{1,2}$ \\ ${ }^{1}$ Imaging Research Laboratories, Robarts Research Institute, \\ London, Ontario, Canada, N6A 5K8 \\ (mwierz, mdrangov, tpeters) @imaging.robarts.ca \\ ${ }^{2}$ Department of Medical Biophysics, The University of Western Ontario, \\ London, Ontario, Canada, N6A 5C1 \\ gguiraud@uwo.ca \\ ${ }^{3}$ Canadian Surgical Technologies \& Advanced Robotics (CSTAR), \\ London, Ontario, Canada, N6A 5A5
}

\begin{abstract}
Currently, minimally invasive cardiac surgery (MICS) faces several limitations, including inadequate training methods using non-realistic models, insufficient surgery planning using 2D images, and the lack of global, 3D guidance during the procedure. To address these issues we are developing the Virtual Cardiac Surgery Platform (VCSP) - a virtual reality model of the patient specific thorax, derived from pre-procedural images. Here we present an image registration-based method for customizing a geometrical template model of the heart to any given patient, and validate it using manual segmentation as the gold standard. On average, the process is accurate to within $3.3 \pm 0.3 \mathrm{~mm}$ in MR images, and $2.4 \pm 0.3 \mathrm{~mm}$ in CT images. These results include inaccuracies in the gold standard, which are on average $1.6 \pm 0.2$ and $0.9 \pm 0.2 \mathrm{~mm}$ for MR and CT images respectively. We believe this method adequately prepares templates for use within VCSP, prior to and during MICS.
\end{abstract}

\section{Introduction}

Coronary artery disease (CAD) and atrial fibrillation (AF) are specific examples of heart disease - the most common cause of death in the developed world. Both conditions can be treated surgically, by strategic scarring of tissues in AF, and by coronary artery bypass grafting in CAD. Conventional surgery is performed via a median sternotomy on the arrested heart, requiring the use of cardiopulmonary bypass (CPB), aortic cross clamping, and myocardial preservation. The latter are the main causes of side effects that lead to increased patient recovery times and costs [1].

Unwanted side-effects, such as large incisions, CPB, or cardiac arrest can be reduced by performing cardiac surgery using an endoscope-aided, port-access approach described previously [2]. In practice however, operating on the beating heart without direct vision is extremely challenging. The first problem is the requirement to perform complex maneuvers, normally carried out in the open chest, inside the closed thoracic cavity. Several systems have been effective for such tasks when used by a trained practitioner [3]. The second and more prominent problem is the lack of proper 
three-dimensional (3D) visualization during the training, planning, and guidance stages of surgery. Currently, only limited training is conducted, usually on unrealistic and expensive animal and cadaver models. Surgery planning of port locations is also inadequate, often based only on 2D images such as x-rays and angiograms. Finally, the current surgical guidance method is imperfect, due to the small field-of-view (FOV) of the endoscope, and the possibility that this view may become obstructed by blood, anatomy, moisture, etc. The lack of proper 3D surgery training, planning, and guidance can lead to improper patient selection, sub-optimal port placement, longer procedures, and increased risks to the patient $[4,5]$.

To address these issues, we continue to develop methods utilizing pre-operative images for 3D modeling of patient anatomy. These methods are combined and utilized in our Virtual Cardiac Surgery Planning (VCSP) platform [6], designed for training, planning, and guidance of minimally invasive cardiac surgeries. In this manuscript, we describe a method for generating patient-specific, geometric models of the heart and its components. Such models are essential in bringing VCSP closer to being an indispensable surgical support tool.

There are two main options for creating the necessary models from pre-operative images: direct segmentation and customization of a template. An example of the direct segmentation approach is the work by Sørensen et al. [7], who created a 3D geometric model of the entire heart from a specialized MR image acquired at enddiastole (ED). Clinical images are often noisy and of low resolution due to imaging time constraints (MR), dose limitations (CT), and unavailability of specialized imaging protocols, making direct segmentation of these images extremely challenging. In addition, it is difficult to use a single segmentation algorithm to detect the various heart structures in multi-modality images required for minimally invasive cardiac surgery. The more suitable method for our application is therefore the template customization approach. An example of this method is the work of LorenzoValdéz, who build template myocardium, LV, and RV models from multiple MR images, and used voxel-based, elastic image registration for customization [8]. Voxel-based methods are well suited for our application, since the results are user independent and the time required per patient is minimal.

In this manuscript, we present our methods for building template models using manual segmentation and for customizing them to target images using a three-stage, image registration algorithm. We perform validation experiments on various MR and CT images, with manual segmentation of the target images acting as the gold standard. Following customization, any given model can be animated using the complete 4D data, as described in our previous work [9]. The final, patient-specific, dynamic model can then be easily integrated with VCSP.

\section{Methods}

In this section we outline our general methods for template-to-patient image mapping and specific methods for algorithm validation. Possible scanning protocols are discussed first, followed by a description of the template model extraction process, and a discussion of the template-to-patient image registration algorithm. 


\subsection{Image Acquisition}

Both MR and CT imaging modalities were considered and tested with our template matching approach. The MR data, consisting of 4D images of six different human volunteers, were acquired with prospective ECG gating, using the $1.5 \mathrm{~T}$, GE CVi scanner, with the fast cine SPGR pulse sequence, an image matrix of $256 \times 128$, eight views per segment, and a 20 degree flip angle. Five of the volunteers were imaged in a clinically practical manner, with two signal averages (NEX) and an average breathhold duration of $23 \mathrm{sec}\left(1.5^{2} \times 6 \mathrm{~mm}^{3}\right.$ voxels). The sixth volunteer was imaged with four NEX, and a breath-hold duration of approximately $40 \mathrm{sec} .\left(1.5^{3} \mathrm{~mm}^{3}\right.$ voxels). The signal-to-noise ratio (SNR) in the higher resolution image was enhanced by registering the four adjacent image frames of the complete 4D data to the image at $\mathrm{ED}$ using the algorithm described in [9], and by averaging the resulting five images. This specialized image is a part of a separate project aimed at generating a dynamic cardiac MR dataset with high resolution and high SNR [10].

The CT data, consisting of four 4D images of canine subjects, were acquired using an eight-slice GE LightSpeed helical CT scanner, with retrospective ECG gating, and $120 \mathrm{kVp}, 200 \mathrm{~mA}$ x-rays. The dogs were paralyzed and artificially ventilated during the $30 \mathrm{sec}$. scan time; one of the dogs was imaged on two different days. Prior to each scan, a bolus of iodine contrast agent $(300 \mathrm{mg} / \mathrm{mL})$ was injected intra-venously to enhance the image contrast inside the heart. The images were reconstructed with a voxel size of $0.35^{2} \times 1.25 \mathrm{~mm}^{3}$.

\subsection{Model Extraction}

Model extraction is normally required once from the ED template image, however, for validation purposes, models of specific parts of the heart anatomy were extracted from each of the ED MR and CT images. A manual, paintbrush technique was used to generate binary data that were then smoothed using a spherical Gaussian filter to remove rough edges. The filtered data were then triangulated using the marching cubes algorithm [11], and finally decimated using a modified version of the algorithm of Schroeder et al. [12] to enable a reasonable rendering speed. For each MR image, the heart was divided into models of the left ventricular myocardium (LVM), right atrium and ventricle (RAV), left atrium and aorta (LAAo), and the epicardial surface (EH). We could only segment the epicardial surface in the canine CT images (EC) due to low contrast inside the heart. Figure 1 shows examples of the resulting models.
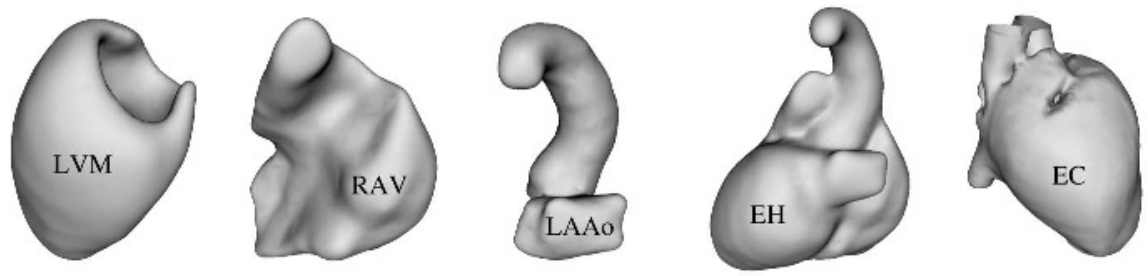

Fig. 1. Left ventricular myocardium (LVM), right atrium and ventricle (RAV), left atrium and aorta (LAAo), and the epicardium (EH) from the higher resolution, human MR image. Also shown is the epicardium obtained from one of the canine CT images (EC). 


\subsection{Registration Algorithm}

For each customization task, the source and target images are selected from the complete 4D data sets so that both represent the heart at approximately the same time point in the cardiac cycle (ED according to the ECG). This reduces differences in morphology between the two hearts, thereby improving our ability to align them accurately. The chosen template image is then customized to the chosen target image using a three-stage registration approach. In the initial stage, the template image is roughly registered with the target image by aligning the two centroids. This is followed by a global registration using an affine transformation, representing translation, rotation, scaling, and shear operations. The 12-degree of freedom (DOF) transformation $\left(\mathrm{T}_{\mathrm{G}}\right)$ can be parameterized in $3 \mathrm{D}$ using the variable $a_{i j}$ as follows:

$$
T_{G}(x, y, z)=\left(\begin{array}{lll}
a_{11} & a_{12} & a_{13} \\
a_{21} & a_{22} & a_{23} \\
a_{31} & a_{32} & a_{33}
\end{array}\right)\left(\begin{array}{l}
x \\
y \\
z
\end{array}\right)+\left(\begin{array}{l}
a_{14} \\
a_{24} \\
a_{34}
\end{array}\right) .
$$

The solution is obtained by a downhill simplex optimization algorithm [13] that maximizes the normalized mutual information (NMI), a similarity metric often used for inter-subject registration [14]. NMI can be expressed as:

$$
\operatorname{NMI}(S, T)=\frac{E_{S}+E_{T}}{2 \cdot E_{S T}},
$$

where $\mathrm{E}_{\mathrm{S}}$ and $\mathrm{E}_{\mathrm{T}}$ are the marginal entropies of the source and target images, and $\mathrm{E}_{\mathrm{ST}}$ is the join entropy. The division by 2 normalizes NMI to lie between 0.5 and 1.0.

Following affine registration, the algorithm begins the non-linear registration stage for local refinement of the template. This step is carried out in a free-form deformation (FFD) framework, where a mesh of control points (nodes) is placed on the template image and then assigned $3 \mathrm{D}$ vectors to indicate local deformation. The result at each node is found by minimizing the following cost function $(\mathrm{C})$ using the downhill simplex method:

$$
C\left(T_{L}^{n}\right)=-N M I\left(S\left(T_{G} T_{L}^{n}, V\right), T(V)\right)+\alpha \cdot B E\left(T_{L}^{n}\right),
$$

where $T_{L}^{n}$ is the translation vector at node $n$ in the FFD grid, $S\left(T_{G}, T_{L}^{n}, V\right)$ is the volume $V$ of the source image surrounding $\mathrm{n}$ after the global transformation $T_{G}$ and local translation $T_{L}^{i}, T(V)$ is the volume $\mathrm{V}$ of the target image surrounding $\mathrm{n}$, and $B E\left(T_{L}^{n}\right)$ is the $3 \mathrm{D}$ thin plate spline bending energy attributed to $T_{L}^{i}$, included to ensure a smooth local transformation (weight controlled by $\alpha$ ) [15]. To reduce the chance of the optimization procedure converging to a local minimum, the algorithm proceeds through three resolution levels of the FFD grid, with the $\mathrm{V}$ parameter progressing from $\sim 7.5$ to 5.0 to $2.5 \mathrm{~cm}^{3}$. Larger $\mathrm{V}$ produces more accurate results since more samples are used to calculate NMI, while smaller V allows more local deformation to be extracted. The highly sampled version of $T_{L}$, required for application to images, is generated using linear interpolation. 


\section{Validation Experiments}

Validation of our template customization algorithm was performed using a single observer, manual segmentation of the target image as the gold standard. Results and discussion are split into two sub-sections, based on the image set analyzed.

\subsection{Human MR Images}

The first task was to quantify the accuracy of the imperfect gold standard itself. For this purpose, an experienced observer manually segmented the LVM, RAV, LAAo, and $\mathrm{EH}$, twice in each of the six images. The intra-observer variability was then quantified as the root mean square (RMS) of the Euclidian distance between a given pair of segmentation surfaces. Correspondence between the surfaces was established by searching for the closest points. Results are in Table 1 below.

Table 1. RMS Euclidian distance between pairs of manually segmented surfaces showing the intra-observer variability of the gold standard. Correspondence was established using nearest points. Image 6 is the high resolution image, not included in the calculation of the mean and standard deviation (SD).

\begin{tabular}{|c|c|c|c|c|}
\hline \multirow{2}{*}{$\begin{array}{c}\text { Data } \\
\text { Compared }\end{array}$} & \multicolumn{4}{|c|}{ RMS (mm) for: } \\
\hline & LVM & RAV & LAAo & EH \\
\hline 1 vs. 1 & 1.85 & 1.97 & 1.95 & 1.71 \\
\hline 2 vs. 2 & 1.36 & 1.39 & 1.69 & 1.39 \\
\hline 3 vs. 3 & 1.50 & 1.35 & 1.46 & 1.49 \\
\hline 4 vs. 4 & 2.06 & 2.14 & 1.74 & 1.53 \\
\hline 5 vs. 5 & 1.37 & 1.56 & 1.11 & 1.16 \\
\hline Mean \pm SD & $1.6 \pm 0.3$ & $1.7 \pm 0.4$ & $1.6 \pm 0.3$ & $1.5 \pm 0.2$ \\
\hline 6 vs. 6 (Hi-res) & 1.35 & 0.79 & 1.22 & 0.95 \\
\hline
\end{tabular}

Table 1 shows that the gold standard can be defined fairly precisely, with the mean RMS error at less than $2 \mathrm{~mm}$. There is no dependence of precision on the anatomy of interest, indicating that inaccuracies arise mostly due to variability in segmenting a particular region, rather than deciding if that region should or should not be segmented. Finally and as expected, we found that the high-resolution image set (image 6 result) could be segmented significantly more precisely than the lower resolution images (mean result).

To determine the accuracy of the customization procedure, one of the two segmentations obtained above for each part of the heart anatomy was randomly selected for every volunteer image. The high-resolution image (6) was then chosen to form the template models, and was registered to each of the remaining images (1 through 5). The error in the customization process was then calculated as the RMS Euclidian distance between the template models, both before and after deformation, and the original target models (Table 2).

Overall, the algorithm was successful at customizing the template models to a given target image. On average $70 \%$ of the unregistered RMS distance was eliminated, with the best results seen in the LVM, due to the high contrast of this structure in MR images. Higher errors of $3.6 \pm 0.4 \mathrm{~mm}$ for the RAV models and $3.5 \pm$ 
$0.3 \mathrm{~mm}$ for the LAAo models are not unexpected due to the $1.5 \times 1.5 \times 6 \mathrm{~mm}^{3}$ voxel size in the target images. Voxel size not only affects the accuracy of the registration, but also the creation of the imperfect "gold standard" model, as seen in Table 1. The errors presented in Table 2 therefore include components that are overestimated (errors calculated with imperfect gold standard) and underestimated (nearest neighbor surface correspondence method).

Table 2. RMS Euclidian distance between the template models before and after customization and the target models. Correspondence was established using nearest points. Unregistered (before customization) results were obtained with models after the centroid alignment stage of registration.

\begin{tabular}{|c|c|c|c|c|c|c|c|c|}
\hline \multirow{2}{*}{$\begin{array}{c}\text { Data } \\
\text { Compared }\end{array}$} & \multicolumn{4}{|c|}{ Unregistered RMS (mm) for: } & \multicolumn{4}{|c|}{ Registered RMS (mm) for: } \\
\hline & LVM & RAV & LAAo & EH & LVM & RAV & LAAo & EH \\
\hline 6 vs. 1 & 7.03 & 8.49 & 9.34 & 8.56 & 3.23 & 3.72 & 3.77 & 3.42 \\
\hline 6 vs. 2 & 9.01 & 11.16 & 8.94 & 9.87 & 3.12 & 3.97 & 3.54 & 3.37 \\
\hline 6 vs. 3 & 11.25 & 13.38 & 17.54 & 15.91 & 2.52 & 3.25 & 3.09 & 2.66 \\
\hline 6 vs. 4 & 8.27 & 10.78 & 10.63 & 10.67 & 2.68 & 3.05 & 3.29 & 3.54 \\
\hline 6 vs. 5 & 10.95 & 12.80 & 13.71 & 13.82 & 2.89 & 4.04 & 3.59 & 3.14 \\
\hline Mean \pm SD & $9.3 \pm 1.8$ & $11.3 \pm 1.9$ & $12.0 \pm 3.6$ & $11.8 \pm 3.0$ & $2.9 \pm 0.3$ & $3.6 \pm 0.4$ & $3.5 \pm 0.3$ & $3.2 \pm 0.4$ \\
\hline
\end{tabular}

\subsection{Canine CT Images}

As for the MR images, the intra-observer variability of the gold standard was quantified by manually segmenting each image twice, and by calculating the RMS Euclidian distance between model pairs. The results are shown in Table 3 below.

Table 3. RMS Euclidian distance between pairs of manually segmented surfaces showing the intra-observer variability of the gold standard. Correspondence was established using nearest points. Image 2 is from the same subject, obtained on two different days.

\begin{tabular}{ccc}
\hline Data Compared & & RMS (mm) for EC \\
\cline { 1 - 1 } 1 vs. 1 & & 0.68 \\
2a vs. 2a & & 1.06 \\
2b vs. 2b & & 0.91 \\
3 vs. 3 & 0.98 \\
\hline Mean \pm SD & & $0.9 \pm 0.2$ \\
\hline
\end{tabular}

Data in Table 3 show a marked improvement in the intra-observer variability obtained with CT images compared to MR. This is most likely due to the almost 88 times increase in resolution of our CT images compared to our MR images, as well as the reduction in breathing motion artefacts achieved in the idealized canine images.

To determine the accuracy of the customization procedure, one of the two segmentations obtained above was randomly selected for each subject. One image was arbitrarily chosen to form the template model and was then registered to each of the remaining images. The error in the customization process was then calculated as the RMS Euclidian distance between the template models, both before and after deformation, and the original target models (Table 4). 
Table 4. RMS Euclidian distance between the template models before and after customization and the target models. Correspondence was established using nearest points. Unregistered (before customization) results were obtained with models after the centroid alignment stage of registration.

\begin{tabular}{|c|c|c|}
\hline $\begin{array}{c}\text { Data } \\
\text { Compared }\end{array}$ & $\begin{array}{c}\text { Unregistered } \\
\text { RMS }(\mathbf{m m}) \text { for EC }\end{array}$ & $\begin{array}{c}\text { Registered } \\
\text { RMS }(\mathbf{m m}) \text { for } \mathrm{EC}\end{array}$ \\
\hline 1 vs. $2 a$ & 6.15 & 2.52 \\
\hline 1 vs. $2 b$ & 6.06 & 2.62 \\
\hline 1 vs. 3 & 6.84 & 2.07 \\
\hline Mean \pm SD & $6.3 \pm 0.4$ & $2.4 \pm 0.3$ \\
\hline
\end{tabular}

The customization procedure with CT images yields models that are significantly more accurate than those obtained with the MR images. Again, note that the error in Table 4 contains components that are overestimated (calculated with imperfect gold standard) and underestimated (nearest neighbor surface correspondence method). The CT data yield better results than MR due to the increased resolution, ideal breath holding, and shorter scan duration.

\section{Conclusions and Future Work}

In this paper we have presented a registration algorithm for mapping template heart models to target images. Validation experiments on human and canine data showed that the method is sufficiently accurate (considering the imperfections in the gold standards) - customizing models with an error on the order of some of the smallest targets for minimally invasive cardiac surgery (coronary arteries). The method was especially successful with CT images, due to the improvement in resolution compared to MR, and the idealized scanning protocol used.

In the future we will use a more robust method of establishing correspondence between two surfaces when calculating registration error. While the nearest point method has been sufficient in this initial study, more sophisticated methods will be required for a thorough investigation. We also plan to perform validation studies on excised hearts, which can easily be segmented if scanned in air, and then surrounded by tissue-mimicking materials to model the heart surroundings in-vivo. Furthermore, we will combine this work and our previous approach [9] to investigate the effects of template registration error on model dynamics, to fully characterize the accuracy of our patient-specific, dynamic models of the heart.

Acknowledgements. The authors thank Aaron So and John Moore for the images, Rhonda Walcarius for MR acquisition, Atamai, Inc. for visualization software, and Ravi Gupta for help with code development. We also acknowledge funding from the Canadian Institutes of Health Research (MOP 14735), Canadian Heart and Stroke Foundation (NA 4755), Ontario Consortium for Image-guided Surgery and Therapy, National Science and Engineering Research Council of Canada, and the University of Western Ontario. 


\section{References}

1. King, R.C., Reece, T.B., Hurst, J.L., Shockley, K.S., Tribble, C.G., Spotnitz, W.D., and Kron, I.L.: Minimally Invasive Coronary Artery Bypass Grafting Decreases Hospital Stay and Cost. Ann Surg 225(6) (1997) 805-811

2. Stevens, J.H., Burdin, T.A., Peters, W.S., Siegel, L.C., Pompili, M.F., Vierra, M.A., St.Goar, F.G., Ribakove, G.H., Mitchell, R.S., and Reitz, B.A.: Port-Access Coronary Artery Bypass Grafting: A Proposed Surgical Method. J Thorac Cardiovasc Surg 111(1996) 567-573

3. Pike, N. and Gundry, S.: Robotically Assisted Cardiac Surgery. J Cardiovasc Nurs 18(5) (2003) 382-388

4. Herzog, C., Dogan, S., Diebold, T., Khan, M.F., Ackermann, H., Schaller, S., Flohr, T.G., Wimmer-Greinecker, G., Moritz, A., and Vogl, T.J.: Multi-Detector Row CT versus Coronary Angiography: Preoperative Evaluation before Totally Endoscopic Coronary Artery Bypass Grafting. Radiology 229(2003) 200-208

5. Boyd, W.D., Desai, N.D., Kiaii, B., Rayman, R., Menkis, A.H., McKenzie, F.N., and Novick, R.J.: A Comparison of Robot-Assisted Versus Manually Constructed Endoscopic Coronary Anastomosis. Ann Thorac Surg 70(2000) 839-843

6. Chiu, A.M., Dey, D., Drangova, M., Boyd, W.D., and Peters, T.M.: 3-D Image Guidance for Minimally Invasive Robotic Coronary Artery Bypass. Heart Surg Forum 3(3) (2000) 224-231

7. Sørensen, T.S., Therkildsen, S., V, Knudsen, J.L., and Pedersen, E.M.: A New Virtual Reality Approach for Planning of Cardiac Intervention. Artif Intell Med 22(3) (2001) 193214

8. Lorenzo-Valdés, M., Sanchez-Ortiz, G., I, Mohiaddin, R., and Ruckert, D.: Atlas-Based Segmentation and Tracking of 3D Cardiac MR Images Using Non-rigid Registration. MICCAI 2002, LNCS 2488(2002) 642-650

9. Wierzbicki, M. and Peters, T.M.: Determining Epicardial Surface Motion Using Elastic Registration: Towards Virtual Reality Guidance of Minimally Invasive Cardiac Interventions. MICCAI 2003, LNCS 2878(2003) 722-729

10. Moore, J., Drangova, M., Wierzbicki, M., Barron, J., and Peters, T.M.: A High Resolution Dynamic Heart Model Based on Averaged MRI Data. MICCAI 2003, LNCS 2878(2003) 549-555

11. Lorensen, W.E. and Cline, H.E.: Marching Cubes: A High Resolution 3D Surface Construction Algorithm. Comput Graph (ACM) 21(4) (1987) 163-169

12. Schroeder, W.J., Zarge, J.A., and Lorensen, W.E.: Decimation of Triangle Meshes. SIGGRAPH 92(26) (1992) 65-70

13. Nelder, J.A. and Mead, R.: A simplex method for function minimization. Computer Journal 7(1965) 308-313

14. Studholme, C., Hill, D.L.G., and Hawkes, D.J.: An overlap invariant entropy measure of 3D medical image alignment. Pattern Recognit 32(1) (1999) 71-86

15. Ruckert, D., Sonoda, L., I, Hayes, C., Hill, D.L.G., Leach, M.O., and Hawkes, D.J.: Nonrigid Registration Using Free-Form Deformations: Application to Breast MR Images. IEEE Trans Med Imaging 18(8) (1999) 712-721 